\title{
Intra-Grain and Oligo-Grain Top-Contact Organic Thin film Transistors
}

Stijn Verlaak, Stijn De Jonge, Stijn Noppe, Dimitri Janssen, Soeren Steudel, Stijn De Vusser and Paul Heremans

Polymer and Molecular Electronics group, IMEC, Kapeldreef 75, B-3001 Leuven, Belgium.

\section{ABSTRACT}

We describe and demonstrate a micromachined shadowmask that allows the realization of intra-grain and oligo-grain top-contact organic thin film transistors (OTFTs). First experimental results of OTFT's show that for small channel lengths, grain boundary barriers indeed appear to dominate the output characteristics of OTFTs.

\section{INTRODUCTION}

Organic thin film transistors (OTFTs) offer a potentially low-cost, large-area technology for low-end applications. Sublimed organic small-molecule thin films offer advantages as active layer in OTFTs compared to solution-processed films. Small-molecule thin films can have higher charge-carrier mobilities due to their crystalline nature and often are of higher purity. However, polycrystalline films have traps at grain boundaries and at the interface with the gate dielectric. Both trap distributions can be expected to depend on growth conditions, providing for a complex relation between morphology and OTFT properties like mobility, threshold voltage, onset voltage and subthreshold slope. Intragrain transistors and transistors having only a limited number of grain boundaries in their channel are a useful tool to study this relation. While photolithographically defined bottom-contacts can easily reach channel lengths comparable to or smaller than the grain size of typical organic thin films, the growth of the organic thin film on top of those contacts is often disturbed by the presence of the contacts. Moreover, contact effects are usually more pronounced than for contacts grown on top of the organic thin films due to the smaller effective contact area of the bottom-contacts [1]. We evaporated metal contacts on top of organic thin films through a micromachined shadowmask to make transistors having channel lengths down to $400 \mathrm{~nm}$. This top-contact approach allows to study the properties of intragrain and oligograin transistors without influencing film growth and with little contact effects. The technology to make those micromachined shadowmask will be presented here, together with the first results on organic thin films.

\section{EXPERIMENT}

The fabrication of the shadowmask using standard micromachining techniques is explained in figure $1.500 \mathrm{~nm} \mathrm{SiO}_{2}$ is grown on a silicon wafer with (100) surface. Next, $150 \mathrm{~nm}$ LPCVD $\mathrm{Si}_{3} \mathrm{~N}_{4}$ is deposited on both sides of the wafer. This nitride will grow almost free of stress on the oxide, apart from some residual tensile stress. On the backside of the wafer large rectangular areas, having a width approximately twice the wafer thickness, are defined approximately parallel to the main crystal axes using photolithography. In those areas, the nitride is removed by $\mathrm{CF}_{4}$ plasma. In a $\mathrm{KOH}$-solution, a trench is etched fully through the silicon wafer selectively along the (111) surfaces, using the nitride on the backside as an etch mask, and the nitride on the frontside as etch stop layer. Finally, the frontside nitride forms a membrane stretched over the 
trench. This membrane can be patterned by various techniques. The easiest way is to protect bridges of nitride stretching over the trench by photolithographically defined patterns of resist and to etch the remaining nitride away in $\mathrm{CF}_{4}$-plasma. The frontside of this mask can be put on an organic film, and metal can be evaporated through the trenches and the holes in the nitride membrane. The nitride bridges will protect the transistor channels. Channel lengths down to 2 $\mu \mathrm{m}$ can be obtained this way. Alternatively, the frontside nitride membrane can be patterned using direct write Focussed Ion Beam (FIB) to locally sputter the nitride away. This technique allows much finer dimensions and more complicated patterns. However, not all patterns are mechanically stable against the intrinsic tensile stress in the nitride. Corners in the pattern need to be rounded off and should not be too close to each other, limiting the minimum width of the transistor channels. We have simulated stress distributions using the finite element method, and designed and implemented stress relief structures in the nitride to guide the stress away from critical dimensions. Channel lengths down to $400 \mathrm{~nm}$ with widths of $10 \mu \mathrm{m}$ were succesfully demonstrated, as well as channel widths of $2 \mu \mathrm{m}$ with channel lengths of $2 \mu \mathrm{m}$. A third alternative, patterning the frontside nitride by e-beam, is under development.
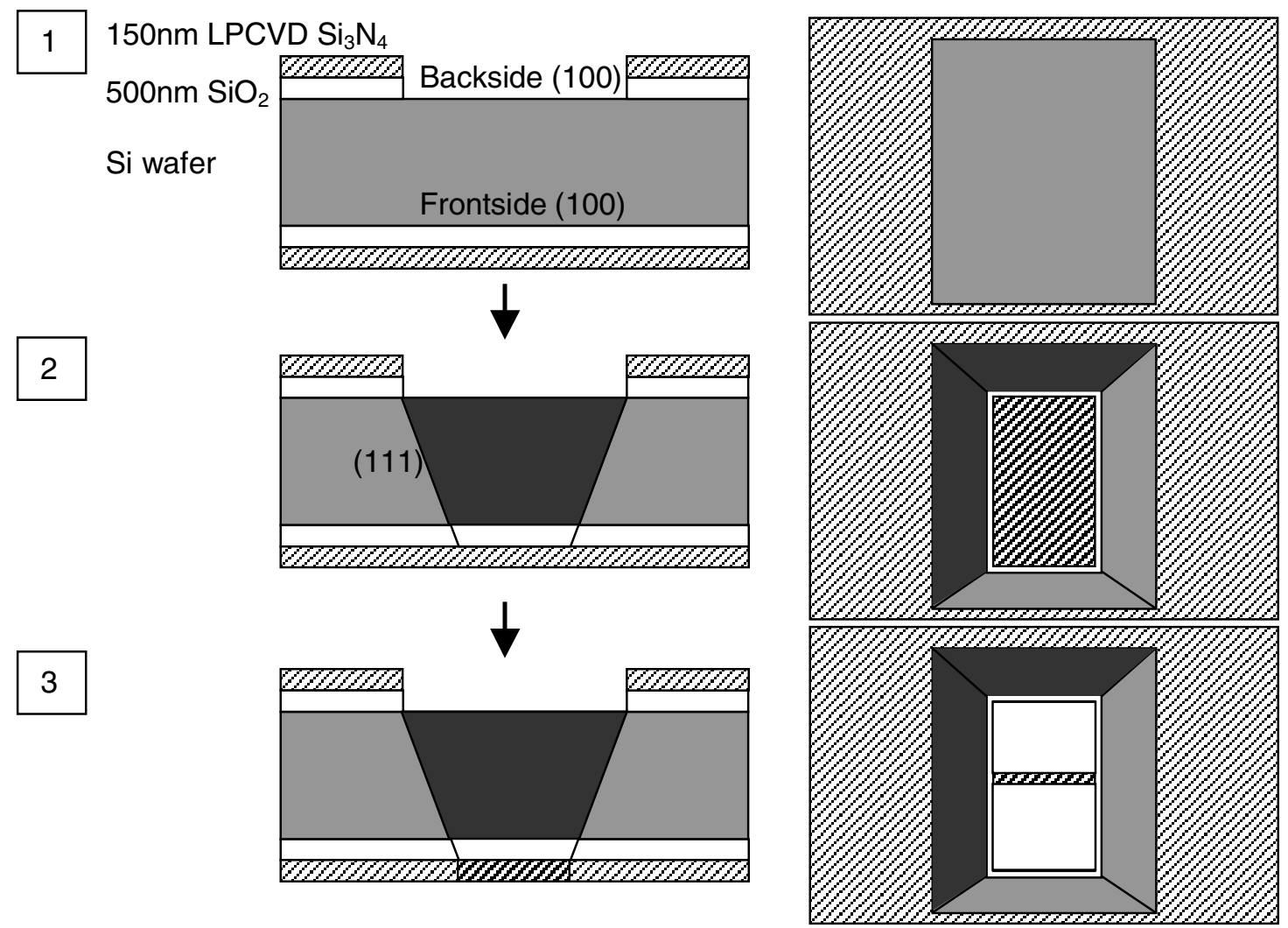

Figure 1. Fabrication of micromachined shadowmask. A (100) silicon wafer is oxidized on both sides, followed by nitride deposition. 1) On the backside of the wafer, the nitride is patterned using photolithography and subsequently etched away in $\mathrm{CF}_{4}$ plasma. 2) $\mathrm{Si}$ is etched away selectively along the (111) planes in a $\mathrm{KOH}$ solution, using the nitride as an etch mask. The $\mathrm{KOH}$ etch stops at the nitride membrane on the front side of the wafer. 3) The nitride membrane can be patterned using Focussed Ion Beam to sputter the nitride away at the desired places, or using lithography followed by another $\mathrm{CF}_{4}$ plasma etch. 

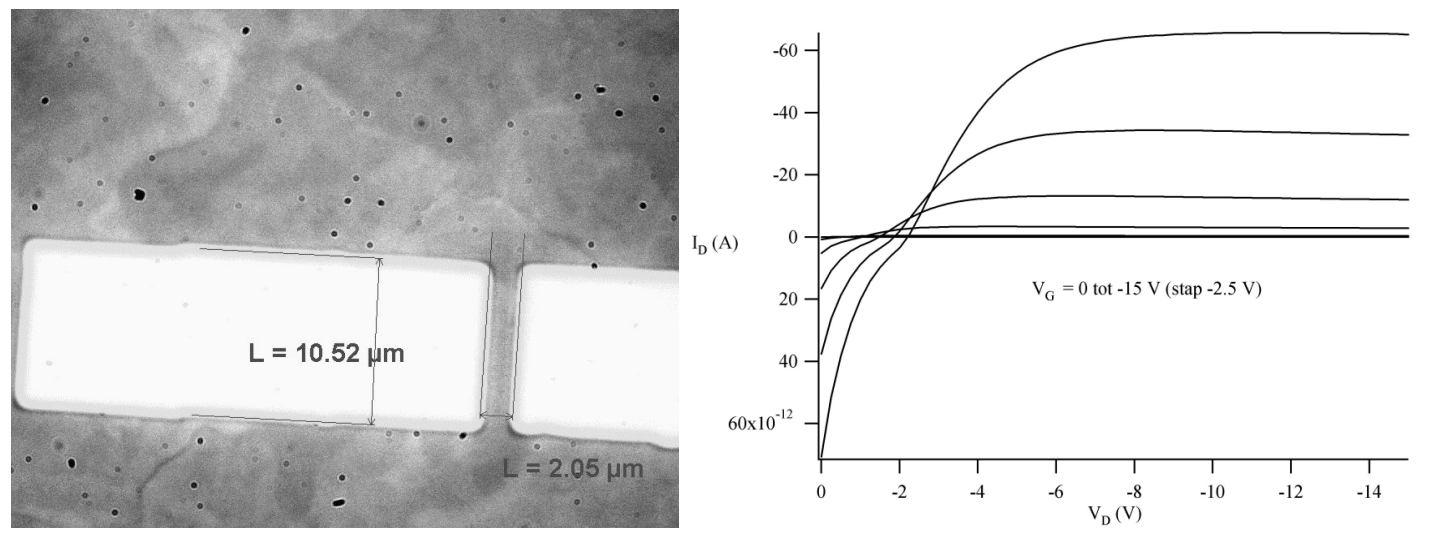

Figure 2. The left figure shows a photograph of a terrylene transistor with a channel length of 2 $\mu \mathrm{m}$. The right figure is the drain current $I_{D}$ versus drain voltage $V_{D}$ characteristics for various gate voltages $\mathrm{V}_{\mathrm{G}}$ for the device in the left picture.

\section{Intra-grain transistors}

To demonstrate this shadowmask technology, intra-grain OTFTs were successfully prepared. Shadowmasks were patterned using FIB, producing W/L's of $2 / 2 \mu \mathrm{m}$ and 10/0.5 $\mu \mathrm{m}$. Organic thin films of terrylene were fabricated on top of $20 \mathrm{~nm}$ thermally grown $\mathrm{SiO}_{2}$ on $\mathrm{Si}$. The substrate functions as gate and gate-dielectric. Terrylene has a relatively high vapor pressure, and therefore more easily allows the growth of thin films with larger grains [2]. Compact grains with diameters of $10 \mu \mathrm{m}$ could be grown. Figure 2 shows an optical picture and the electrical characteristics of one terrylene transistor with shadowmask evaporated gold contacts. The terrylene transistor had $\mathrm{W} / \mathrm{L}=10 \mu \mathrm{m} / 2 \mu \mathrm{m}$, and the extracted mobility is $\sim 10^{-5} \mathrm{~cm}^{2} / \mathrm{Vs}$. This low mobility is attributed to the large amount of impurities as indicated by TOFSIMS analysis of the thin film. No clear transistor behaviour could be distinguished in devices with three or more grain boundaries, even with larger W/L. Although the FIB does not allow to easily make many devices nor many shadowmasks, and the terrylene material lacks electronic grade purity, those devices clearly demonstrate that this shadowmask technology is functional. No shorts were detected between source and drain for channel lengths down to $0,5 \mu \mathrm{m}$.

\section{$\underline{\text { Oligo-grain transistors }}$}

Using photolithographically patterned shadowmasks, transistors could be made with channel lengths L varying between 2 and $50 \mu \mathrm{m}$, while the channel width is determined by the width of trench etched in the silicon wafer, typically varying between 30 and $150 \mu \mathrm{m}$. Pentacene thin films were grown on octadecyltrichlorosilane (OTS) treated surfaces of 100nm thermally oxidized highly doped silicon wafers. The oxidized wafers again function as gate and gate-dielectric. On top of the pentacene film, source and drain contacts with varying channel lengths were deposited. Figure 3 shows an AFM picture of the pentacene film, together with the $I_{D}-V_{D}$ characteristics of transistors with various channel lengths. It can clearly be seen that the drain current does not saturate for the smallest channel, while the saturation improves for increasing channel lengths. For the given channel lengths and oxide thickness, the lack of saturation cannot be explained by a deviation from the gradual channel approximation. We tentatively attribute this to drain induced grain boundary barrier effects, which show similar characteristics [3]. 

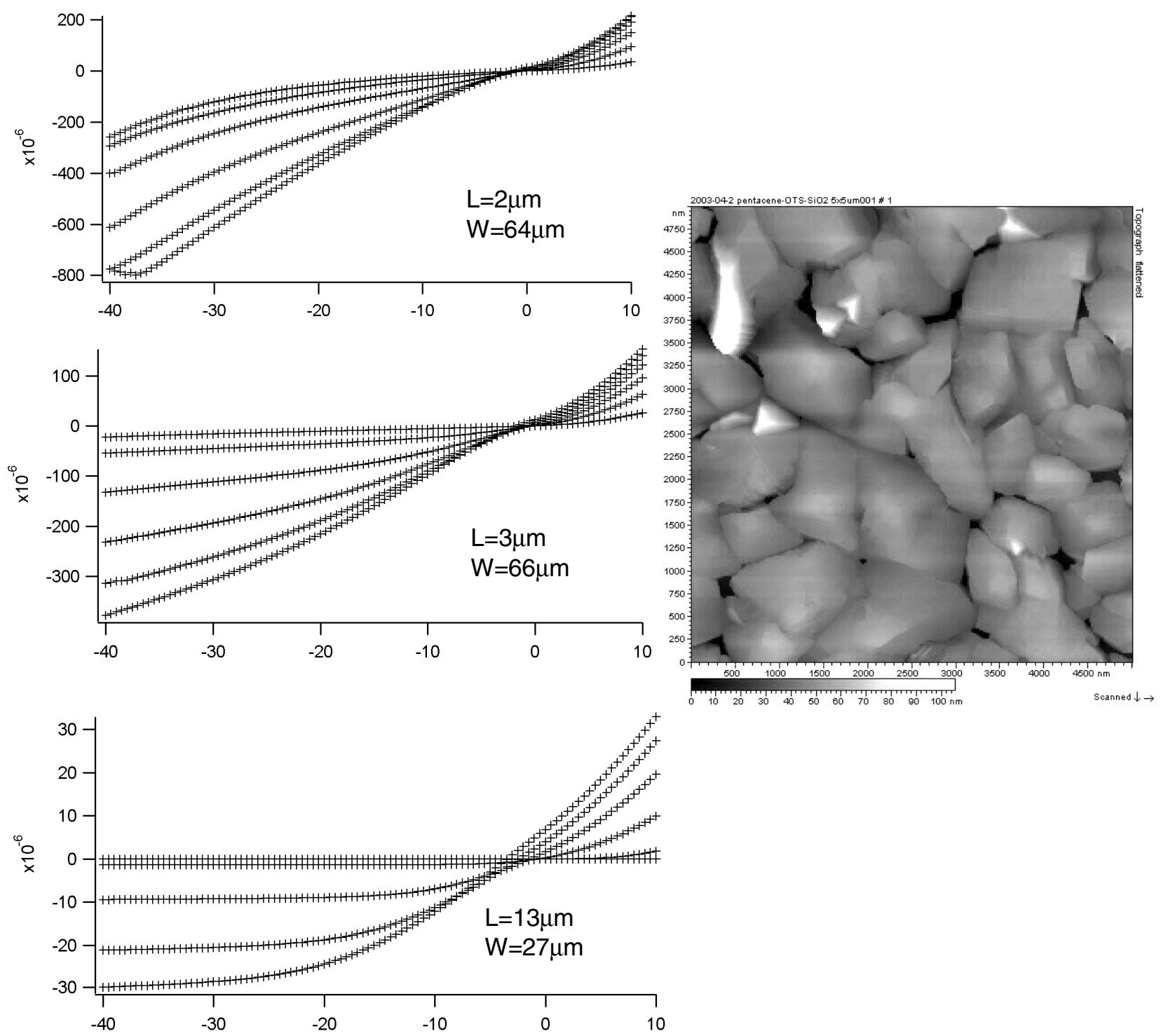

Figure 3. Pentacene transistors with channel lengths of 2, 3 and $13 \mu \mathrm{m}$. The gate voltage varies between $+10 \mathrm{~V}$ to $-40 \mathrm{~V}$ in steps of $10 \mathrm{~V}$. The saturation regime improves noticeably with increasing channel length. The electrical characteristics are dominated by grain boundary barriers

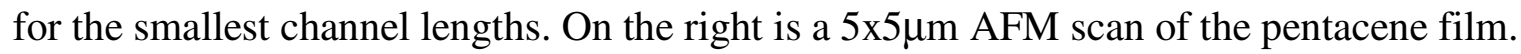

\section{CONCLUSIONS}

We have developed and demonstrated a micromachined shadowmask that is used to fabricate short-channel OTFTs, which allow for probing intra-grain and grain boundary effects. Intra-grain devices offer the opportunity to extract the intrinsic charge-carrier mobility, yet also magnify various device-related issues. Oligo-grain devices appear to be dominated by grain boundary barrier effects. 


\section{ACKNOWLEDGEMENTS}

We like to acknowledge Agnes Verbist for her input in the processing technology, Steve Reyntjens for operating the FIB, prof. Klaus Mullen and Erik Reuther for providing a sample of terrylene, and prof. Norbert Karl for interesting discussions.

\section{REFERENCES}

1. R.A. Street and A. Salleo, Appl. Phys. Lett. 81, 2887-2889 (2002).

2. S. Verlaak, S. Steudel, D. Janssen, P. Heremans and M. Deleuze, to be published.

3. C.A. Dimitriadis and D.H. Tassis, J. Appl. Phys. 77, 2177-2183 (1995). 